\title{
NON-ARCHIMEDEAN ENTIRE CURVES IN CLOSED SUBVARIETIES OF SEMI-ABELIAN VARIETIES
}

\author{
JACKSON S. MORROW
}

\begin{abstract}
We prove a non-archimedean analogue of the fact that a closed subvariety of a semiabelian variety is hyperbolic modulo its special locus, and thereby generalize a result of Cherry.
\end{abstract}

\section{INTRODUCTION}

The Green-Griffiths-Lang-Vojta conjectures predict that a quasi-projective variety $X$ over $\mathbb{C}$ is of log-general type if and only if there is a proper closed subscheme $\Delta \subset X$ such that $X$ is Brody hyperbolic modulo $\Delta$ (i.e., every non-constant holomorphic map $\mathbb{C} \rightarrow X(\mathbb{C})$ factors through $\Delta(\mathbb{C})$ ); see [Bro17, BD18, GG80, Lan86, Voj15, Rou18]. For example, this conjecture is known when $X$ is a closed subvariety of an abelian variety by the celebrated theorem of Bloch-Ochiai-Kawamata [Blo26, Och77, Kaw80]. We refer the reader to [NWY07, NWY08] for more recent advances.

The aim of this paper is to investigate non-archimedean analogues of the Green-Griffiths-LangVojta conjectures. Our starting point is the following theorem, which is the culmination of results in [Fa191, Fal94, Abr94, Voj96, Nog98]. The definitions of the notions appearing in the following theorem are stated in [Lan87, p. 78] and [Jav19, Definitions 7.1, 8.1].

Theorem 1.1 (Abramovich, Faltings, Kawamata, Noguchi, Ueno, Vojta). Let X be a closed subvariety of a semi-abelian variety $\mathrm{G}$ over $\mathbb{C}$. Let $\mathrm{Sp}(\mathrm{X})$ be the union of the subvarieties of $\mathrm{X}$ which are translates of positive-dimensional closed subgroups of $\mathrm{G}$. Then the following statements hold.

(1) The subset $\mathrm{Sp}(\mathrm{X})$ is Zariski closed in $\mathrm{X}$.

(2) The variety $X$ is of log-general type if and only if $\operatorname{Sp}(X) \neq X$.

(3) The variety $X$ is arithmetically hyperbolic modulo $\mathrm{Sp}(\mathrm{X})$.

(4) The variety $X$ is Brody hyperbolic modulo $\mathrm{Sp}(\mathrm{X})$.

In [Che94, Che96, CR04, ACW08, LW10, LW17], the authors investigate possible non-archimedean analogues of the Green-Griffiths-Lang conjecture; however, some of their results contrast the complex analytic setting. Inspired by Cherry's work, the authors of [JV18] formulated the "correct" analogue of the Green-Griffiths-Lang conjecture for projective varieties over a non-archimedean valued field $\mathrm{K}$.

Our main result is the non-archimedean analogue of the statements (2), (3), and (4) in Theorem 1.1. We refer the reader to Section 2 for the definition of a K-analytic Brody hyperbolic variety.

Theorem A. Let $\mathrm{K}$ be an algebraically closed complete non-archimedean valued field of characteristic zero. Let $\mathrm{X}$ be a closed subvariety of a semi-abelian variety $\mathrm{G}$ over $\mathrm{K}$. Then X is K-analytically Brody hyperbolic modulo $\operatorname{Sp}(X)$.

This result is proven by Cherry [Che94, Theorem 3.5] when G is an abelian variety (so that $X$ is projective). Our line of reasoning to prove Theorem A resembles Cherry's in that we study analytic morphisms from tori to semi-abelian varieties (see Section 3).

2010 Mathematics Subject Classification. 32H20, (32P05).

Key words and phrases. Semi-abelian varieties, hyperbolicity, Lang-Vojta conjecture, rigid analytic varieties, nonarchimedean geometry, Albanese variety, p-adic uniformization. 
A direct consequence of Theorem $\mathrm{A}$ is the following characterization of groupless ([JK19, Definition 2.1]) closed subvarieties of a semi-abelian variety.

Corollary B. Let $\mathrm{K}$ be an algebraically closed complete non-archimedean valued field of characteristic zero, and let $\mathrm{X}$ be a closed subvariety of a semi-abelian variety $\mathrm{G}$ over $\mathrm{K}$. Then $\mathrm{X}$ is groupless (i.e., does not contain the translate of a positive-dimensional closed subgroup of $\mathrm{G}$ ) if and only if $\mathrm{X}$ is $\mathrm{K}$-analytically Brody hyperbolic.

Acknowledgments. The author would like to thank Ariyan Javanpeykar for suggesting the problem, for many helpful conversations, and for sending a preliminary version of [Jav19], which helped in writing Section 2. The author extends his thanks to Alberto Vezzani and David ZureickBrown for useful discussions. The author also thanks Lea Beneish for comments on an earlier draft.

Conventions. Throughout, $\mathrm{K}$ denotes an algebraically closed complete non-archimedean valued field of characteristic zero. For a locally of finite type scheme $X / K$, we will use $X^{\text {an }}$ to denote the rigid analytic space (in the sense of Tate [Tat71]) or the adic space (in the sense of Huber [Hub94]) associated to $X$, unless otherwise stated.

\section{NON-ARCHIMEDEAN HYPERBOLICITY OF PAIRS}

In this section, we extend the notion of K-analytic Brody hyperbolicity of varieties introduced in [JV18]. First, we recall their notion.

Definition 2.1 ([JV18, Definition 2.3, Lemma 2.14, Lemma 2.15]). Let $X$ be a finite type separated scheme over K. Then X is K-analytically Brody hyperbolic if

- every analytic morphism $G_{m, K}^{a n} \rightarrow X^{\text {an }}$ is constant, and

- for every abelian variety $A$ over $K$, every morphism $A \rightarrow X$ is constant.

We now define what it means for a pair $(X, D)$ to be hyperbolic. Our proposed definition reads as follows.

Definition 2.2. Let $X$ be a finite type separated scheme over $K$ and let $D \subset X$ be a closed subscheme. Then X is K-analytically Brody hyperbolic modulo D (or: the pair (X, D) is K-analytically Brody hyperbolic) if

- every non-constant analytic morphism $G_{m, K}^{\mathrm{an}} \rightarrow X^{\text {an }}$ factors over $D^{\text {an }}$, and

- for every abelian variety $A$ over $K$ and every dense open subset $U \subset A$ with $\operatorname{codim}(A \backslash$ $\mathrm{U}) \geqslant 2$, every non-constant morphism $\mathrm{U} \rightarrow \mathrm{X}$ of schemes factors over $\mathrm{D}$.

With this definition, it is not hard to see that a proper scheme $\mathrm{X}$ over $\mathrm{K}$ is $\mathrm{K}$-analytically Brody hyperbolic if and only if $(X, \varnothing)$ is $\mathrm{K}$-analytically Brody hyperbolic (i.e., $X$ is K-analytically Brody hyperbolic modulo the empty set). Indeed, if $X$ is K-analytically Brody hyperbolic and proper, then $X$ has no rational curves. In particular, every rational map $A \rightarrow X$ with $A$ an abelian variety extends (uniquely) to a morphism $A \rightarrow X$, and such morphisms are constant if $X$ is $K$-analytically Brody hyperbolic.

Similarly, one can show that a closed subscheme $X$ of a semi-abelian variety is K-analytically Brody hyperbolic if and only if $(X, \varnothing)$ is K-analytically Brody hyperbolic; see Remark 2.4.

Remark 2.3. The reader might find the condition in Definition 2.2 on the codimension of the complement of $\mathrm{U}$ in $\mathrm{A}$ unnatural. We now explain why this condition is necessary (assuming one wants to define the "right" notion of hyperbolicity). Note that Vojta has already made the observation that one has to test hyperbolicity on "big" open subsets of algebraic groups and not merely on algebraic groups; see [Voj15, Definition 2.2] and also [Jav19, §6]. 
The example to keep in mind is the blow-up $X$ of a simple abelian surface $A$ at the origin over $Q$. It is not hard to see that $X(\mathbb{C})$ admits a dense entire curve, and is therefore as far as possible from being Brody hyperbolic (in the usual complex-analytic sense).

Let $p$ be a prime of good reduction of $A$, and consider the smooth projective variety $X_{C_{p}}$ over $\mathbb{C}_{p}$. Let $\Delta$ be the exceptional locus of $X_{\mathbb{C}_{p}} \rightarrow A_{C_{p}}$. Then, every non-constant morphism from $\mathbb{G}_{m}^{a n} \rightarrow X_{\mathbb{C}_{p}}^{\text {an }}$ factors over $\Delta^{\text {an }}$. Moreover, by rigid analytic GAGA [Köp74] and the simplicity of $A_{C_{p}}$, for every abelian variety $B$ over $C_{p}$, every morphism $B^{a n} \rightarrow X_{C_{p}}^{a n}$ is constant. Thus, if one does not "test" the hyperbolicity on big opens of abelian varieties, the variety $X_{\mathbb{C}_{p}}$ would be $\mathbb{C}_{p^{-}}$ analytically Brody hyperbolic modulo $\Delta$ (contrary to it being very far from being Brody hyperbolic over $\mathbb{C}$ ).

Remark 2.4. Let $A / K$ be an abelian variety and let $G / K$ be a semi-abelian variety. By [Moc12, Lemma A.2], for every dense open subset $U \subset A$ with $\operatorname{codim}(A \backslash U) \geqslant 2$, we have that any morphism $U \rightarrow G$ extends uniquely to a morphism $A \rightarrow G$. Using this result, we immediately have that a closed subscheme $X$ of $G$ is $\mathrm{K}$-analytically Brody hyperbolic if and only if $\mathrm{X}$ is $\mathrm{K}$ analytically Brody modulo $\varnothing$.

Definition 2.5. A finite type separated scheme $X$ over $\mathrm{K}$ is pseudo-K-analytically Brody hyperbolic if there is a proper closed subset $\mathrm{D} \subsetneq \mathrm{X}$ of $\mathrm{X}$ such that $(X, D)$ is $\mathrm{K}$-analytically Brody hyperbolic.

\section{ANALYTIC MAPS FROM TORI TO SEMI-ABELIAN VARIETIES}

Let $G$ be a semi-abelian variety over $K$. Since $G$ is semi-abelian, there is a split torus $T_{1} \subset G$, an abelian variety $A$ over $K$, and a short exact sequence of commutative group schemes

$$
0 \rightarrow \mathrm{T}_{1} \rightarrow \mathrm{G} \rightarrow \mathrm{A} \rightarrow 0 .
$$

Our goal is to prove that, if $\phi: \mathbb{G}_{m}^{a n} \rightarrow G^{\text {an }}$ is a morphism, then the Zariski closure of its image is the translate of the analytification of an algebraic subgroup of $\mathrm{G}$; see Proposition 3.6 for a precise statement.

Remark 3.1. In this section, for a locally of finite type scheme $X / K$, we will use $X^{\text {an }}$ to denote the associated K-analytic space (in the sense of Berkovich [Ber90]). We do so in order to use techniques from topology to study analytic maps from tori to semi-abelian varieties. In particular, an analytic torus is simply-connected [Ber90, Section 6.3], and a famous result of Berkovich [Ber99, Corollary 9.5] states that a smooth, connected, Hausdorff strictly K-analytic space has a universal covering which is a Hausdorff, simply connected strictly K-analytic space.

We can relate our results concerning Berkovich spaces to adic spaces using the equivalence between the category of Hausdorff strictly K-analytic spaces and the category of taut locally of finite type adic spaces [Hub96, Proposition 8.3.7].

We start by recalling that line bundles on analytifications of tori are trivial.

Lemma 3.2. Let $X$ be a separated, good, strictly $\mathrm{K}$-analytic space, and let $\mathrm{X}_{0}$ denote the associated rigid analytic space. Then $\operatorname{Pic}(X) \cong \operatorname{Pic}\left(X_{0}\right)$.

Proof. This follows from [Ber93, Corollary 1.3.5] and the bottom of loc. cit. p. 37.

Lemma 3.3. If $\mathrm{L}$ is a line bundle on a split torus $\mathrm{G}_{\mathrm{m}, \mathrm{K}}^{\mathrm{r}, \mathrm{an}}$, then $\mathrm{L}$ is trivial.

Proof. Since the Berkovich analytification of $\mathbb{G}_{m, K}^{r}$ is a separated, good, strictly K-analytic space, our result follows from [FvdP04, Theorem 6.3.3.(2)] and Lemma 3.2.

Lemma 3.4. Let $\phi: \mathbb{G}_{\mathrm{m}}^{\mathrm{an}} \rightarrow \mathrm{G}^{\mathrm{an}}$ be a morphism, and let $\widetilde{\phi}: \mathbb{G}_{\mathrm{m}}^{\mathrm{an}} \rightarrow \widetilde{\mathrm{G}}$ be a lift of this morphism to the universal cover $\widetilde{\mathrm{G}}$ of $\mathrm{G}^{\mathrm{an}}$. Then, the image $\widetilde{\phi}\left(\mathbb{G}_{\mathrm{m}}^{\mathrm{an}}\right)$ is contained inside a split torus $\mathrm{T}^{\mathrm{an}}$ of $\widetilde{\mathrm{G}}$. 
Proof. Let $\widetilde{A}$ be the universal covering of $A^{\text {an }}$. By [BL84, Uniformization Theorem 8.8], there is a semi-abelian variety $H$ over $K$ with $\widetilde{A} \cong H^{\text {an }}$, an abelian variety $B$ over $K$ with good reduction over $\mathcal{O}_{\mathrm{K}}$, a split torus $\mathrm{T}_{2} \subset \mathrm{H}$, and a short exact sequence of commutative group schemes

$$
0 \rightarrow \mathrm{T}_{2} \rightarrow \mathrm{H} \rightarrow \mathrm{B} \rightarrow 0
$$

Let $\widetilde{G}$ be the universal covering space of $G^{\text {an }}$. Note that there is a structure of a commutative Berkovich analytic group on $\widetilde{\mathrm{G}}$ which makes $\widetilde{\mathrm{G}} \rightarrow \mathrm{G}^{\text {an }}$ into a homomorphism. By the universal property of universal covering spaces, the surjective homomorphism $G^{\text {an }} \rightarrow A^{\text {an }}$ lifts uniquely to a homomorphism $\widetilde{\mathrm{G}} \rightarrow \mathrm{H}^{\text {an }}$.

The image of $\mathbb{G}_{m}^{a n} \rightarrow \widetilde{G} \rightarrow H^{\text {an }}$ is contained in $T_{2}^{a n}$. Indeed, the morphism $\mathbb{G}_{m}^{\text {an }} \rightarrow H^{\text {an }} \rightarrow B^{\text {an }}$ is constant, since $B^{\text {an }}$ has good reduction [Che94, Theorem 3.2], and so the image of $G_{m}^{\text {an }}$ in $H^{\text {an }}$ lands inside its torus $\mathrm{T}_{2}^{\text {an }}$ (up to translation).

Since $T_{1}^{\text {an }}$ is simply-connected [Ber90, Section 6.3], the subgroup $T_{1}^{\text {an }} \subset G^{\text {an }}$ lifts to a subgroup $\mathrm{T}_{1}^{\text {an }} \subset \widetilde{\mathrm{G}}$. Note that the homomorphism $\mathrm{T}_{1}^{\mathrm{an}} \rightarrow \mathrm{H}^{\mathrm{an}}$ factors over $\mathrm{T}_{2}^{\mathrm{an}}$, and that the morphism $\mathrm{T}_{1}^{\mathrm{an}} \rightarrow \mathrm{T}_{2}^{\mathrm{an}}$ is algebraic [Che94, Proposition 3.4], i.e., the analytification of some morphism $\mathrm{T}_{1} \rightarrow \mathrm{T}_{2}$. Let $T_{3}$ be the image of this morphism, which is again a split torus.

Let $F$ be the inverse image of $T_{3}^{a n} \subset H^{\text {an }}$ in $\widetilde{G}$. Note that $F$ is a closed subgroup of $\widetilde{G}$ and that the kernel of the homomorphism $\mathrm{F} \rightarrow \mathrm{T}_{3}^{\text {an }}$ equals $\mathrm{T}_{1}^{\mathrm{an}}$. Thus, there is a short exact sequence of rigid analytic groups

$$
0 \rightarrow \mathrm{T}_{1}^{\mathrm{an}} \rightarrow \mathrm{F} \rightarrow \mathrm{T}_{3}^{\text {an }} \rightarrow 0
$$

By Lemma 3.3, the above sequence splits, and so $F$ is the analytification of a split torus $T$. This shows that the image $\widetilde{\phi}\left(\mathbb{G}_{m}^{\text {an }}\right)$ is contained inside the split torus $T^{\text {an }}$, as required.

Lemma 3.5. Let $\phi: \mathbb{G}_{m}^{a n} \rightarrow G^{\text {an }}$ be a morphism. Suppose that the image of $\mathbb{G}_{m}^{a n} \rightarrow G^{\text {an }} \rightarrow A^{\text {an }}$ is Zariski dense. Then, the image of $\phi$ is Zariski dense in $\mathrm{G}^{\text {an }}$.

Proof. Lemma 3.4 asserts that $\phi\left(\mathbb{G}_{m}^{\mathrm{an}}\right)$ is an analytic subgroup $\mathrm{F}^{\prime}$ of $\mathrm{G}^{\mathrm{an}}$, as it is the composition of group homomorphisms $\widetilde{\phi}$ and the uniformization map, which is an analytic group homomorphism. Since $G_{m}^{a n} \rightarrow G^{\text {an }} \rightarrow A^{\text {an }}$ is Zariski dense, $F^{\prime}$ dominates $A^{\text {an }}$, and this analytic group homomorphism has kernel $\mathrm{T}_{1}^{\mathrm{an}}$. Moreover, we have the following morphism of short exact sequences of analytic groups:

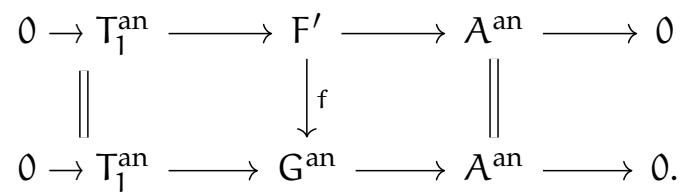

By Berkovich analytic GAGA [Ber90, Corollary 3.4.10], $\operatorname{Pic}\left(A^{\text {an }}\right)$ is in bijective correspondence with $\operatorname{Pic}(A)$, which implies that $F^{\prime}$ is in fact an algebraic subgroup of $G^{\text {an }}$. Moreover, the short five lemma tells us that the morphism $f$ must be an isomorphism.

Proposition 3.6. Let $\phi: \mathbb{G}_{m}^{\mathrm{an}} \rightarrow \mathrm{G}^{\mathrm{an}}$ be a morphism. Then, the Zariski closure of $\phi\left(\mathbb{G}_{\mathrm{m}}^{\mathrm{an}}\right)$ in $\mathrm{G}^{\mathrm{an}}$ is the analytification of the translate of of an algebraic subgroup of $\mathrm{G}$.

Proof. Let $\psi: \mathbb{G}_{\mathrm{m}}^{\mathrm{an}} \rightarrow \mathrm{A}^{\text {an }}$ be the composition of $\phi$ and the surjective homomorphism $G^{\text {an }} \rightarrow A^{\text {an }}$. By Lemma 3.4, the image $\phi\left(\mathbb{G}_{m}^{\mathrm{an}}\right)$ is an analytic subgroup of $G^{\text {an }}$. Therefore, the image $\psi\left(\mathbb{G}_{m}^{\text {an }}\right)$ is an analytic subgroup of $A^{\text {an }}$. Thus, the Zariski closure of the image of $\psi$ is an abelian subvariety $E^{\text {an }}$ of $A^{\text {an }}$ (see [Che94, Proof of Theorem 3.6]).

Now, let $F$ be the preimage of $E$ inside $G$, and note that $F$ is a semi-abelian variety (as it is a closed subgroup of $G$ ). Clearly, the image of the morphism $\phi: \mathbb{G}_{m}^{\text {an }} \rightarrow G^{\text {an }}$ is contained in $F^{\text {an }}$. Now, by construction, the image of the composed morphism $\mathbb{G}_{m}^{a n} \rightarrow F^{\text {an }} \rightarrow E^{\text {an }}$ is Zariski dense 
in E. Therefore, by Lemma 3.5, the image of $G_{m}^{a n}$ in $F^{a n}$ is the analytification of the translate of an algebraic subgroup of $F$. In particular, it is the analytification of the translate of an algebraic subgroup of $\mathrm{G}$.

The following example shows that the image of an algebraic group under an analytic homomorphism is not necessarily an algebraic subgroup.

Example 3.7. Let $E / K$ be an elliptic curve with multiplicative reduction and let $\phi: \mathbb{G}_{m, K}^{a n} \rightarrow E^{\text {an }}$ be the universal covering of $E^{a n}$. Consider the semi-abelian variety $G=G_{m, K} \times E$. Let $G_{m, K}^{a n} \rightarrow$ $\mathrm{G}_{\mathrm{m}, \mathrm{K}}^{\mathrm{an}} \times \mathrm{E}^{\mathrm{an}}$ be the morphism defined by $z \mapsto(z, \phi(z))$. The image of this morphism is not an algebraic subgroup of $G^{\text {an }}$. However, its Zariski closure equals $G^{\text {an }}$.

To end this section, we prove Theorem A.

Proof of Theorem A. Proposition 3.6 tells us that the Zariski closure of every analytic morphism $\mathrm{G}_{m}^{\mathrm{an}} \rightarrow X^{\text {an }} \subset \mathrm{G}^{\text {an }}$ is contained in $\operatorname{Sp}(X)^{\text {an }}$. To conclude the proof, it suffices to show that for every abelian variety $A$ over $K$ and every dense open subset $U \subset A$ with $\operatorname{codim}(A \backslash U) \geqslant 2$, we have that every non-constant morphism $U \rightarrow X$ of schemes factors over $\operatorname{Sp}(X)$. By Remark 2.4, every morphism $\mathrm{U} \rightarrow \mathrm{X} \subset \mathrm{G}$ extends to a morphism $A \rightarrow X \subset \mathrm{G}$. Now, by [Iit76, Theorem 2], any morphism between semi-abelian varieties is the composition of a group homomorphism and a translation, so that the image of $A \rightarrow X \subset G$ factors over $\operatorname{Sp}(X)$, as desired.

\section{REFERENCES}

[Abr94] Dan Abramovich. Subvarieties of semiabelian varieties. Compositio Math., 90(1):37-52, 1994.

[ACW08] Ta Thi Hoai An, W. Cherry, and Julie Tzu-Yueh Wang. Algebraic degeneracy of non-Archimedean analytic maps. Indag. Math. (N.S.), 19(3):481-492, 2008.

[BD18] Damian Brotbek and Lionel Darondeau. Complete intersection varieties with ample cotangent bundles. Invent. Math., 212(3):913-940, 2018.

[Ber90] Vladimir G. Berkovich. Spectral theory and analytic geometry over non-Archimedean fields, volume 33 of Mathematical Surveys and Monographs. American Mathematical Society, Providence, RI, 1990.

[Ber93] Vladimir G. Berkovich. Étale cohomology for non-Archimedean analytic spaces. Inst. Hautes Études Sci. Publ. Math., (78):5-161 (1994), 1993.

[Ber99] Vladimir G. Berkovich. Smooth p-adic analytic spaces are locally contractible. Invent. Math., 137(1):1-84, 1999.

[BL84] Siegfried Bosch and Werner Lütkebohmert. Stable reduction and uniformization of abelian varieties. II. Invent. Math., 78(2):257-297, 1984.

[Blo26] André Bloch. Sur les systèmes de fonctions uniformes satisfaisant è l'équation d'une variété algébrique dont l'irrégularité dépasse la dimension. J. Math. Pures Appl., 5:9-66, 1926.

[Bro17] Damian Brotbek. On the hyperbolicity of general hypersurfaces. Publ. Math. Inst. Hautes Études Sci., 126:1-34, 2017.

[Che94] W. Cherry. Non-Archimedean analytic curves in abelian varieties. Math. Ann., 300(3):393-404, 1994.

[Che96] William Cherry. A non-Archimedean analogue of the Kobayashi semi-distance and its non-degeneracy on abelian varieties. Illinois J. Math., 40(1):123-140, 1996.

[CR04] William Cherry and Min Ru. Rigid analytic Picard theorems. Amer. J. Math., 126(4):873-889, 2004.

[Fal91] Gerd Faltings. Diophantine approximation on abelian varieties. Ann. of Math. (2), 133(3):549-576, 1991.

[Fal94] Gerd Faltings. The general case of S. Lang's conjecture. In Barsotti Symposium in Algebraic Geometry (Abano Terme, 1991), volume 15 of Perspect. Math., pages 175-182. Academic Press, San Diego, CA, 1994.

[FvdP04] Jean Fresnel and Marius van der Put. Rigid analytic geometry and its applications, volume 218 of Progress in Mathematics. Birkhäuser Boston, Inc., Boston, MA, 2004.

[GG80] Mark Green and Phillip Griffiths. Two applications of algebraic geometry to entire holomorphic mappings. In The Chern Symposium 1979 (Proc. Internat. Sympos., Berkeley, Calif., 1979), pages 41-74. Springer, New YorkBerlin, 1980.

[Hub94] Roland Huber. A generalization of formal schemes and rigid analytic varieties. Math. Z., 217(4):513-551, 1994.

[Hub96] Roland Huber. Étale cohomology of rigid analytic varieties and adic spaces. Aspects of Mathematics, E30. Friedr. Vieweg \& Sohn, Braunschweig, 1996.

[Iit76] Shigeru Iitaka. Logarithmic forms of algebraic varieties. J. Fac. Sci. Univ. Tokyo Sect. IA Math., 23(3):525-544, 1976. 
[Jav19] A. Javanpeykar. The Lang-Vojta conjectures on projective pseudo-hyperbolic varieties. In preparation, 2019.

[JK19] A. Javanpeykar and L. Kamenova. Demailly's notion of algebraic hyperbolicity: geometricity, endomorphisms, moduli of maps. arXiv:1807.03665, January 17, 2019.

[JV18] A. Javanpeykar and A. Vezzani. Non-archimedean hyperbolicity and applications. arXiv:1808.09880, August 29, 2018.

[Kaw80] Yujiro Kawamata. On Bloch's conjecture. Invent. Math., 57(1):97-100, 1980.

[Köp74] Ursula Köpf. Über eigentliche Familien algebraischer Varietäten über affinoiden Räumen. Schr. Math. Inst. Univ. Münster (2), (Heft 7):iv+72, 1974.

[Lan86] Serge Lang. Hyperbolic and diophantine analysis. Bull. Amer. Math. Soc. (N.S.), 14:159-205, 1986.

[Lan87] Serge Lang. Introduction to complex hyperbolic spaces. Springer-Verlag, New York, 1987.

[LW10] Chien-Wei Lin and Julie Tzu-Yueh Wang. Generalizations of rigid analytic Picard theorems. Proc. Amer. Math. Soc., 138(1):133-139, 2010.

[LW17] Aaron Levin and Julie Tzu-Yueh Wang. On non-Archimedean curves omitting few components and their arithmetic analogues. Canad. J. Math., 69(1):130-142, 2017.

[Moc12] Shinichi Mochizuki. Topics in absolute anabelian geometry I: generalities. J. Math. Sci. Univ. Tokyo, 19(2):139242, 2012.

[Nog98] Junjiro Noguchi. On holomorphic curves in semi-abelian varieties. Math. Z., 228(4):713-721, 1998.

[NWY07] Junjiro Noguchi, Jörg Winkelmann, and Katsutoshi Yamanoi. Degeneracy of holomorphic curves into algebraic varieties. J. Math. Pures Appl. (9), 88(3):293-306, 2007.

[NWY08] Junjiro Noguchi, Jörg Winkelmann, and Katsutoshi Yamanoi. The second main theorem for holomorphic curves into semi-abelian varieties. II. Forum Math., 20(3):469-503, 2008.

[Och77] Takushiro Ochiai. On holomorphic curves in algebraic varieties with ample irregularity. Invent. Math., 43(1):83-96, 1977.

[Rou18] Erwan Rousseau. KAWA lecture notes on complex hyperbolic geometry. Ann. Fac. Sci. Toulouse Math. (6), 27(2):421-443, 2018.

[Tat71] John Tate. Rigid analytic spaces. Invent. Math., 12:257-289, 1971.

[Voj96] Paul Vojta. Integral points on subvarieties of semiabelian varieties. I. Invent. Math., 126(1):133-181, 1996.

[Voj15] Paul Vojta. A Lang exceptional set for integral points. In Geometry and analysis on manifolds, volume 308 of Progr. Math., pages 177-207. Birkhäuser/Springer, Cham, 2015.

Jackson S. Morrow, Department of Mathematics, Emory University, Atlanta, GA 30322, United STATES

E-mail address: jmorrow4692@gmail.com 